\title{
Influência dos parâmetros de síntese da cerâmica supercondutora do tipo $\mathrm{SmBaCuO}$
}

\author{
Capucho, I.M. ${ }^{1}$; Passos, C. A. C. ${ }^{1}$; Orlando, M. T. D. ${ }^{1}$; Abílio, V.T. ${ }^{2}$; Cruz, P. C. M. ${ }^{2}$ \\ 1 Programa de Pós-Graduação em Engenharia Mecânica, Universidade Federal do Espírito Santo, Vitória, ES, Brasil \\ 2 Departamento de Física, Universidade Federal do Espírito Santo, Vitória, ES, Brasil
}

\begin{abstract}
Resumo
Neste trabalho foi preparado amostras policristalinas de $\mathrm{SmBa}_{2} \mathrm{Cu}_{3} \mathrm{O}_{7}-\mathrm{d}$ (Sm-123) com diferentes parâmetros de síntese com finalidade de relacionar a influência no parâmetro de rede, tamanho de grão e temperatura crítica (Tc). As amostras foram preparadas pela técnica de reação sólido-vapor a partir dos seguintes reagentes: $0,5 \mathrm{Sm}_{2} \mathrm{O}_{3}+3 \mathrm{CuO}+2 \mathrm{BaCO}_{3}$. As amostras foram rotuladas de Amostra A e Amostra B. A oxigenação foi feita em $520{ }^{\circ} \mathrm{C}$ por 24 horas. Além disto, estas amostras foram caracterizadas por Difração de Raios X (DRX), Microscopia Eletrônica de Varredura (MEV) e Susceptibilidade Magnética ac. Os resultados de DRX indicam que a três fases presentes: $\mathrm{Sm}-123$ otorrômbico, $\mathrm{Sm}-123$ tetragonal e $\mathrm{BaCuO}_{2}+\mathrm{x}$. Além disso, dos difratogramas foram determinados os parâmetros de rede para cada amostra. A partir das análises das micrografias de MEV foram obtidos o tamanho médio das junções de grãos cujos valores foram 1,64 $\mu \mathrm{m}$ e 2,6 $\mu \mathrm{m}$ respectivamente. Por fim, os resultados da susceptibilidade magnética revelaram que $\mathrm{TC}_{\mathrm{A}}=91 \mathrm{~K}, \mathrm{TC}_{\mathrm{B}}=92 \mathrm{~K}$. Concluímos que de acordo com a síntese aplicada os parâmetros utilizados se modificam, mas não alteram significativamente a temperatura crítica.
\end{abstract}

Palavras chaves: Tamanho de Grão, Parâmetro de Rede, Temperatura Crítica, Difração de Raios X.

\section{Introdução}

Desde a descoberta dos supercondutores de alta Tc, permanece uma expectativa para desenvolvimento de dispositivos para aplicações tecnológicas devido aos avanços nos processos de sínteses dos cupratos [1,2]. No entanto, as cerâmicas impõem limites para tais dispositivos devido às propriedades dos grãos da cerâmica [3]. Assim, qualquer que seja a aplicação, é crucial controlar os processos de fabricação das amostras cerâmicas. Além disso, determinar as propriedades granulares e correlacionar com os parâmetros de síntese dos compostos supercondutores policristalinos.

\section{Síntese do Supercondutor}

A preparação das amostras foram feitas a partir de uma reação de estado sólido utilizando os óxidos de cobre ( $\mathrm{CuO}$ ) e samário $\left(\mathrm{Sm}_{2} \mathrm{O}_{3}\right)$ juntamente com o carbonato de bário $\left(\mathrm{BaCO}_{3}\right)$. A reação seguiu a seguinte proporção de $0,5 \mathrm{~mol}$ de $\mathrm{Sm}_{2} \mathrm{O}_{3}, 2$ moles de $\mathrm{BaCO}_{3}$ e 3 moles de $\mathrm{CuO}$. As amostras foram misturadas, prensadas e pastilhadas, em seguida, levadas ao forno para a preparação do precursor. O tratamento térmico do precursor seguiu o patamar de $40 \mathrm{~h}$ a uma temperatura de $960^{\circ} \mathrm{C}$ à atmosfera ambiente [4].
Tabela 1: Processo de síntese de cada amostra supercondutora.

\begin{tabular}{ccc}
\hline Amostras & A & B \\
\hline $\begin{array}{c}\text { Taxa de aquecimento } \\
\left({ }^{\circ} \mathrm{C} / \mathrm{min}\right)\end{array}$ & 10 & 10 (até $\left.900^{\circ} \mathrm{C}\right) \mathrm{e}$ \\
depois 2 até $\left(1060^{\circ} \mathrm{C}\right)$ \\
\hline $\begin{array}{c}\text { Temperatura de } \\
\text { Síntese }\left({ }^{\circ} \mathrm{C}\right)\end{array}$ & 1060 & 1060 \\
\hline
\end{tabular}

Em seguida, repetiu-se o procedimento e as amostras foram recolocadas ao forno para o processo de síntese. Este processo de síntese teve parâmetros distintos conforme descrito na Tabela 1 e com controle de fluxo de oxigênio de 30 bolhas por minuto. Em seguida, as amostras foram então caracterizadas por Difração de Raios X, Microscopia Eletrônica de Varredura e Susceptibilidade Magnética ac.

\section{Resultados e Discussão}

Os resultados da difração de raios $X$ (Fig. 1) revelaram que há mistura de fases, onde aparecem as estruturas cristalinas otorrômbica e tetragonal. Para a amostra A há $91 \%$ de fase otorrômbica, $6 \%$ de fase tetragonal e $2 \%$ de resídua de $\mathrm{BaCuO}_{2+x}$ e $1 \%$ de picos não identificados. No caso da amostra B $89 \%$ de otorrômbica, $7 \%$ de tetragonal, $2 \%$ de $\mathrm{BaCuO}_{2+x}$ e $2 \%$ 
de picos não identificados. Além disso, foram obtidos os parâmetros de rede similares que estão resumidos na Tabela 2. Os resultados foram obtidos através do programa CELREF [5].

Tabela 2: Parâmetros de rede da fase otorrômbica e temperatura crítica das amostras supercondutoras.

\begin{tabular}{|c|c|c|}
\hline Parâmetros & Amostra A & Amostra B \\
\hline 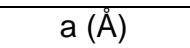 & $3,903(2)$ & $3,903(2)$ \\
\hline $\mathrm{b}(\AA)$ & $3,844(1)$ & $3,844(1)$ \\
\hline c $(\AA)$ & $11,725(3)$ & $11,724(1)$ \\
\hline Tc $(\mathrm{K})$ & $91,0(1)$ & $92,3(1)$ \\
\hline
\end{tabular}

Estas amostras também foram analisadas por Microscopia Eletrônica de Varredura (MEV). A partir da imagens de MEV e com auxílio do programa ImageJ [6], foram contabilizados tamanho de junções dos grãos. Os dados foram dispostos em histogramas e analisados com auxílio da função densidade de distribuição gama para se obter o tamanho médio das junções. Para a amostra A foi obtido 2,05(2) $\mu \mathrm{m}$ e para amotra B 1,49(2) $\mu \mathrm{m}$.

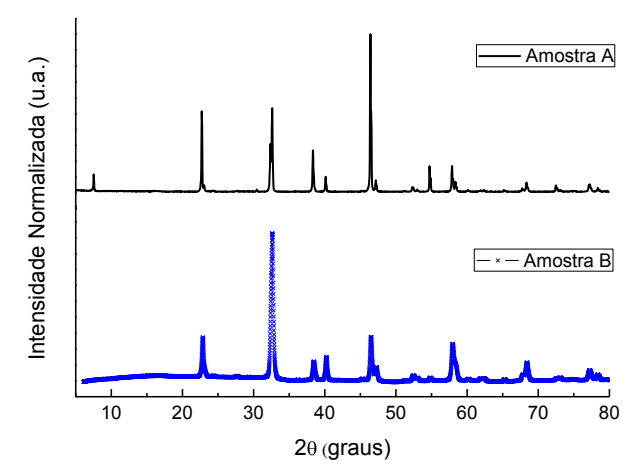

Figura 1: Padrão de difração de raios $X$ para amostra $A$ e para amostra B.

Por fim estas amostras foram submetidas a susceptibilidade magnética $\mathrm{AC}(\chi a c)$, e os resultados são apresentados na Figura 4. Tc valores foram determinados pelo critério da segunda derivada da susceptibilidade. Os resultados mostram que cada uma das amostras tem blindagem magnética distintas, o que estar relacionado com o tamanho médio de grão. Isto sugere que o processo de síntese afetou a quantidade de oxigenação das amostras.

\section{Conclusão}

Amostras policristalinas de $\mathrm{SmBa}_{2} \mathrm{Cu}_{3} \mathrm{O}_{7-d}$ com diferentes parâmetros de síntese foram produzidas pela técnica de reação de estado sólido. Os resultados de difração de raios $X$ revelaram que as mostras são um mistura de fase, sendo que fase supercondutora é predominante. Além disso, o procedimento de síntese utilizado neste trabalho influenciou no crescimento dos grão. Assim amostra A apresentou maior tamanho médio de junções e maior blindagem magnética.
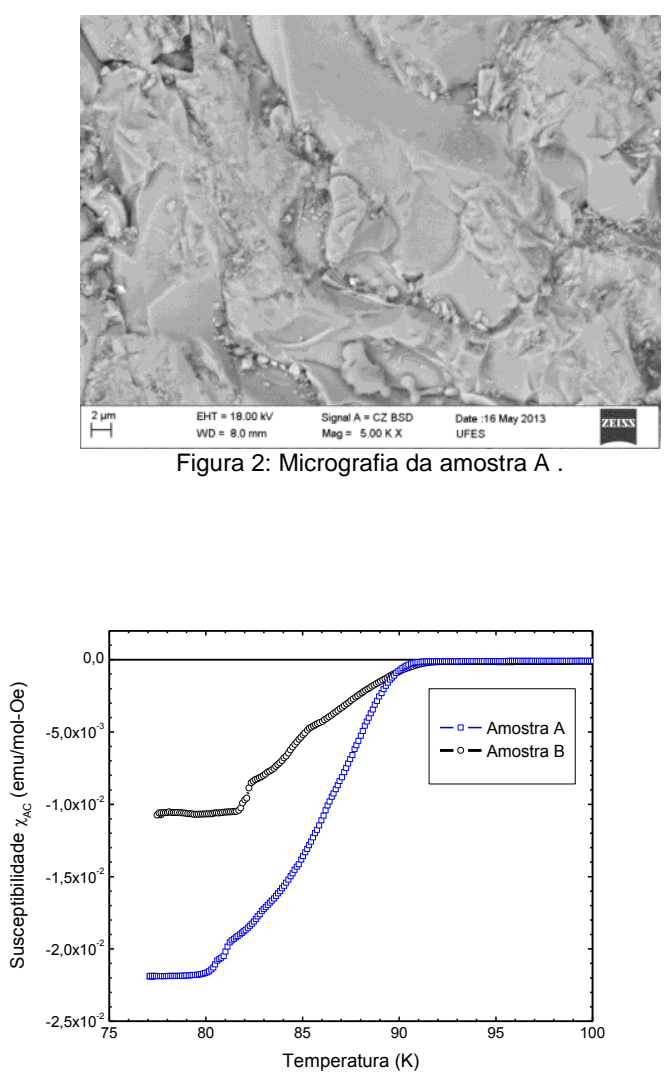

Figura 3: Comparação das medida de susceptibilidade ac em função da temperatura.

\section{Agradecimentos}

Os autores agradecem as agências de fomento CAPES e FAPES.

\section{Referências}

[1] NOUDEM, J.G.; BOURGAULT, D.; BARBUT, J.M.; TIXADOR, P.; TOURNIER, R. Phys. C, v. 349, p. 4752, 2001.

[2] HARI BABU, N.; LIDA, K.; SHI, Y.; CARDWELL, D.A. Phys. C, v. 468, p.1340-1344, 2008.

[3] PASSOS, C.A.C.; ORLANDO, M.T.D.; KELLER, G. L. L.; PASSAMAI Jr., J. L.; FERREIRA, J. A.; MELLO, E. V. L. Phys. B, v. 404 , 3123 -3126, 2009.

[4] MAÑKA, J.; CIGÁÑ, A.; POLOVKOVÁ, J.; KOÑAKOVSKY, A.; PRNOVÁ, A.; Measurement Science Review, v. 11, p. 9-14, 2011.

[5] Software de refinamento dos parâmetros de rede, Celref. Disponível em <http://amin.janghorban.perso.sfr.fr/download.htm>. Acesso em: 12 Mai. 2014.

[6] Informações e download do programa ImageJ. Disponível em <http://rsb.info.nih.gov/ij/docs/index.html >. Acesso em: 12 Mai. 2014. 